\title{
Circulating tumor DNA analysis of metastatic renal cell carcinoma
}

\author{
JINGBO ZHANG ${ }^{1}$, YUNCHAO LIU $^{1}$, BING XU ${ }^{1}$, FUWEI LI $^{1}$, YAN WANG ${ }^{1}$, MENGJIAN LI ${ }^{1}$, \\ RONG DU $^{1}$, YE ZHOU ${ }^{1}$, MEGHAN SALGIA ${ }^{2}$, LIXIN YANG ${ }^{2}$ and JEREMY O. JONES ${ }^{2}$ \\ ${ }^{1}$ Beijing USCI Medical Laboratory, Beijing 100049, P.R. China; ${ }^{2}$ Department of Medical Oncology, \\ City of Hope Medical Center, Duarte, CA 91010, USA
}

Received June 8, 2020; Accepted October 13, 2020

DOI: $10.3892 / \mathrm{mco} .2020 .2178$

\begin{abstract}
The genomic landscape of metastatic renal cell carcinoma (RCC) is not well understood, and currently available data suggest that it is functionally distinct from that of localized tumors. Additionally, the large number of approved and trial agents used to treat metastatic RCC likely cause selective adaptations in the tumors. Circulating tumor DNA (ctDNA) is a platform to non-invasively determine the genomic profiles of these tumors. The objectives of the present study were to corroborate previous ctDNA studies in metastatic RCC, to identify novel mutations in metastatic $\mathrm{RCC}$, and to compare ctDNA profiles obtained from plasma and urine in patients with metastatic RCC. ctDNA sequencing using the plasma and urine of 50 patients with metastatic RCC who received ctDNA profiling as part of routine clinical care at a single institution was performed using an investigational 120-gene panel. Genomic alterations (GAs) were identified in all 50 patients. The genes with the most GAs were GNAS, PTEN, MYC, MET and HNF1A and novel mutations in additional genes were identified. A significant correlation between the number of GAs detected in matched urine and plasma samples was also identified, but only $28.1 \%$ of GAs detected in plasma samples were also detected in matched urine samples. The results of the present study were consistent with those of the largest previous study of ctDNA from patients with metastatic RCC and may help identify additional potential targets for the treatment of such patients.
\end{abstract}

\section{Introduction}

Renal cell carcinoma ( RCC) is the seventh most common type of cancer worldwide, and its incidence in different countries, including Canada, Saudi Arabia and Belarus, is on the increase (1). The 5-year overall survival (OS) is $\sim 74 \%$, although $\sim 30 \%$ of patients with RCC who present with evidence of distant metastasis upon initial diagnosis have a 5-year OS of

Correspondence to: Dr Jeremy O. Jones, Department of Medical Oncology, City of Hope Medical Center, 1500 East Duarte Road, Duarte, CA 91010, USA

E-mail: jorionjones@gmail.com

Key words: metastasis, renal cell carcinoma, circulating tumor DNA only $8 \%$ (2). Despite the fact that $>10$ agents have now been approved for the treatment of metastatic RCC, the survival rates have not improved drastically. This may be attributed to the fact that these agents are often used without guided biomarkers that may be used to ensure that the most effective treatments are administered to selected patients. Thus, there is a need to further elucidate the genomic landscape of metastatic RCC and to identify biomarkers for guided therapy.

Due to the valuable contributions of The Cancer Genome Atlas (TCGA) and other researchers $(3,4)$, there is currently a clear picture of the genomic landscape of several RCCs; however, nearly all the samples from these studies were derived from primary nephrectomies and from earlier stages of disease. It is likely that the genomic landscape of metastatic RCCs differs from than that of primary RCCs, as the cancers may evolve in more advanced stages and through selective pressures from treatment. While repeat biopsies of metastatic lesions are challenging due to financial and medical reasons, circulating tumor DNA (ctDNA) offers the opportunity to assess genomic alterations in metastatic cancers. This approach has been used in a number of different malignancies to identify changes in tumors over time (5). Indeed, a recent investigation of ctDNA from metastatic RCC samples demonstrated clear differences from the genomic landscape of primary RCC and an evolution of genomic alterations (GAs) under treatment with targeted agents (6). In this cohort of 220 patients, high frequencies of GAs were identified in TP53, VHL1, EGFR, NF1, as well as several other genes. A number of these genes had more frequent GAs in samples from patients with multiple lines of therapy vs. those receiving only one line of therapy. However, the genomics of metastatic ctDNA have yet to be fully elucidated. Furthermore, with the plethora of treatment options and the presence of rapidly evolving tumors, there is a need to identify biomarkers to individualize treatment in order to ensure maximum efficacy. Therefore, the aim of the present study was to determine whether the patterns of GAs in our cohort were consistent with previous findings and to further elucidate the genomics of metastatic RCC in patients with varied treatment patterns using a more comprehensive ctDNA sequencing panel.

\section{Materials and methods}

Sample collection and processing. As part of the IRB-approved study 17089, patients included in the present series had a diagnosis of metastatic RCC and consented to blood and urine 
collection for ctDNA assessment as a part of routine clinical care at City of Hope Medical Center (Duarte, USA). Urine was collected in a standard collection cup and frozen within $2 \mathrm{~h}$. Blood was collected in Cell-Free DNA BCT collection tubes (Streck). To separate plasma, whole blood was centrifuged at $300 \mathrm{x}$ g for $20 \mathrm{~min}$ at room temperature. The upper plasma layer was removed and transferred to a new conical tube which was then centrifuged at 5,000 $\mathrm{x}$ g for $10 \mathrm{~min}$ at $20^{\circ} \mathrm{C}$. Cell-free DNA (cfDNA) was isolated using the QIAamp Circulating Nucleic Acid Kit (Qiagen, Inc.); the median collection amount was $359.7 \mathrm{ng}$ (range, 222.4-1,329.7 $\mathrm{ng}$ ) for plasma and 2,111.4 $\mathrm{ng}$ (range, 456.9-3,101.8 $\mathrm{ng}$ ) for urine. cfDNA was shipped on dry ice to Beijing Scisoon Biotechnology Ltd., Co. for analysis. The concentration of cfDNA was determined using Qubit fluorometer 3.0 (Thermo Fisher Scientific, Inc.), KAPA Hyper Prep Kit (KK8504, Roche Diagnostics) was used for library construction. Library quality control was measured using 2100 Bioanalyzer (Agilent Technologies, Inc.) and then 150 base pair-end sequencing was performed on Illumina Novaseq 6000 (Illumina, Inc.). Reads were aligned to human reference genome GRCh37/hg19 using BWA (v0.7.17; https://github.com/lh3/bwa) and single-nucleotide variants were determined by VarScan (v2.4.1; https://github. com/dkoboldt/varscan). The sequenced genes are shown in Table SI.

Statistical analysis. The mean depth of sequencing coverage by sample had a mean \pm SD of 2,979.514x $\pm 1,420.744$ (range, 1,008.54-6,353.28x) for plasma and 2,756.0 $\pm 3,338.112$ (range, 361.4-14,180.0) for urine. The frequency of GAs was assessed in the overall cohort and compared across line of therapy and histology (when available). Multivariable regression was used to evaluate the associations among clinical variables and GAs. The frequencies of individual GAs in subgroup analyses were compared using $\chi^{2}$ tests. $\mathrm{P}<0.05$ was considered to indicate statistically significant differences.

\section{Results}

Patient characteristics and association with distribution of GAs. ctDNA was collected from the urine and plasma from a total of 50 patients with mRCC between June 2018 and December 2018 (Table I). The median age of the cohort was 65 years (range, $36-89$ years), and the patients included 40 men and 10 women. Samples were collected from patients with varying therapies, lines of treatment and histological subtypes (Tables I and SII). Using an investigational ctDNA panel that covers part or the entirety of 120 genes (Table SI), it was observed that all 50 plasma samples contained GAs compared to the consensus sequences at these loci (median GA no. 2; range, 1-6). The number of GAs was significantly associated with the number of lines of therapy, both on univariate regression analysis $(\mathrm{P}=0.038)$ and on multivariable regression analysis $(\mathrm{P}=0.047)$, which included age, sex, $\mathrm{T}$ stage, Fuhrman grade, and histological subtype; no other variables were associated with the number of GAs (Tables SIII and SIV).

The genes with the most frequent GAs were GNAS, PTEN, $M Y C, M E T$ and HNF1A (Fig. 1A). Full lists of GAs found in plasma samples are in provided in Table SV. Our findings bear
Table I. Patient characteristics.

\begin{tabular}{lr} 
Clinical variables & Patient \\
\hline Sex & \\
Male & 40 \\
Female & 10 \\
Lines of therapy & \\
0 & 1 \\
1 & 28 \\
2 & 9 \\
3 & 6 \\
$4+$ & 6 \\
T stage & \\
1 & 9 \\
2 & 12 \\
3 & 22 \\
4 & 2 \\
Unknown & 5
\end{tabular}

Fuhrman grade

1

2

3

4

40

10

1

28

9

6

6

12

22

5

grade 0

$\begin{array}{ll}7 & 7\end{array}$

Unknown 6

Histology

Clear cell $\quad 40$

$\begin{array}{ll}\text { Papillary } & 7\end{array}$

Chromophobe 3

some similarities to the largest published analysis of ctDNA from $\mathrm{mRCC}$ patients to date (6), despite using different sequencing panels (Table II). A similar high frequency of GAs was observed in $M E T$ and $M Y C$, and a similar low frequency of GAs in RET, JAK3, IDH1 and AKT1. Pal et al (6) reported a higher frequency of GAs in TP53 compared with that of the present study, while we identified a higher frequency of GAs in GNAS and PTEN. Additional GAs were identified in RICTOR, FGFR4, AURKA, MYD 88 and FLT1, none of which were sequenced by $\mathrm{Pal}$ et al.

Pal et al (6) also observed significant increases in the number of patients with GAs in TP53, VHL, EGFR, NF1 and $P I K 3 C A$ among patients who had received multiple lines of therapy vs. those receiving only first-line therapy. We searched for increased GAs in patients who had multiple lines of therapy $(n=22)$ vs. those with only first-line therapy $(n=28)$; however, the only significant difference identified by $\chi^{2}$ analysis was an increased frequency of GAs in GNAS in patients with only first-line therapy $(\mathrm{P}=0.025)$. Site of metastasis data were available on $43 / 50$ patients in our database. A total of 7 patients had multiple metastatic sites, and the overall involvement included 21 lymph node, 31 lung, 2 brain, 4 liver, 7 bone and 9 'other soft tissue' lesions. Subgroup analysis by metastatic site for enrichment in GAs in PTEN, MYC, GNAS or MET did not identify any significant differences by $\chi^{2}$ analyses (Table SVI). 

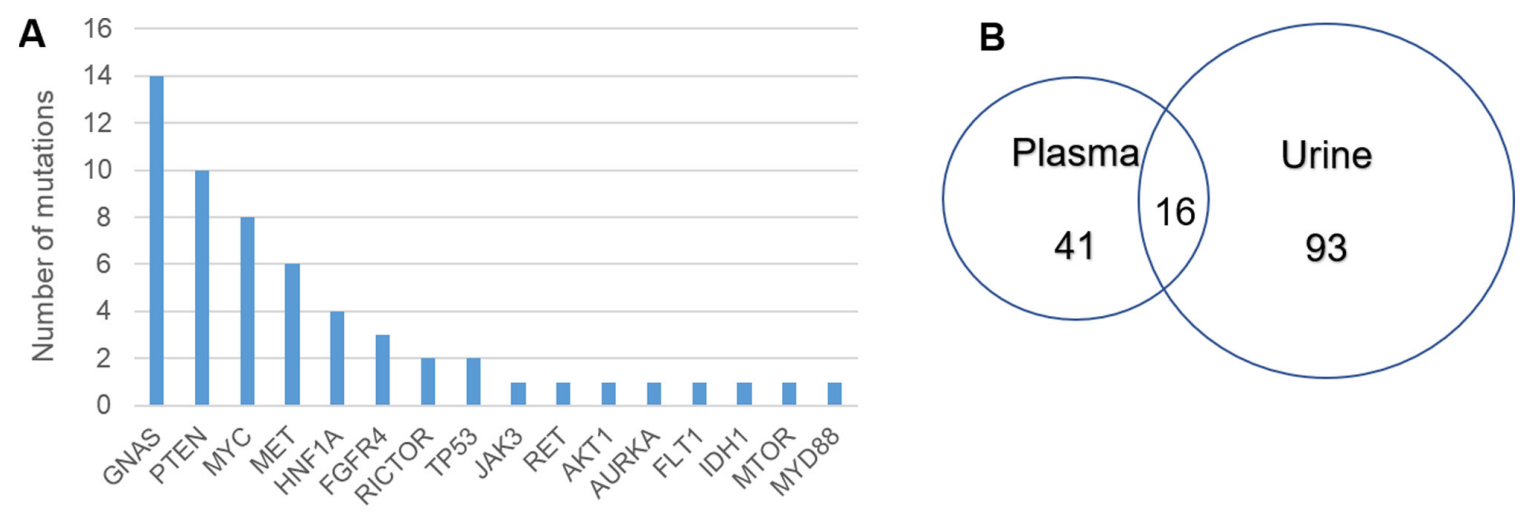

Figure 1. (A) Number of plasmid samples ( $\mathrm{n}=50)$ with GAs in the indicated genes. (B) Venn diagram showing GAs detected in matched plasma and urine samples. GAs, genomic alterations.

Matched urine samples were also obtained from the patients, 45 of which yielded ctDNA sequencing results that passed quality control filters. All 45 samples had at least one GA (median, 3; range, 1-8). Regression analysis demonstrated a significant association between the number of GAs detected in matched plasma and urine samples $(\mathrm{P}=0.047)$. While several GAs were unique to urine samples (Fig. 1B), 28.1\% (16/57) of the GAs detected in plasma samples were also detected in matched urine samples.

\section{Discussion}

In the largest assessment of ctDNA from metastatic RCC patients to date, $\mathrm{Pal}$ et al (6) reported high rates of GAs in several genes, including TP53, VHL, EGFR, PIK3CA and NF1, and that the rate of GAs in these genes was higher in patients receiving multiple lines of therapy vs. first-line therapy. The present study, although from a smaller cohort, identified several similarities to this previous study, with high frequencies of mutations in MET and MYC. There were also several differences, including higher GA frequencies in the GNAS and PTEN genes, and several GAs we identified in genes that were not sequenced in the previous study, including RICTOR, FGFR4, AURKA, MYD 88 and FLT1.

GNAS was the most frequently mutated gene in our cohort, with the majority of mutations occurring at two residues. The A448D mutation has been previously identified in prostate and pancreatic adenocarcinomas (7), while, to the best of our knowledge, the mutation at aa 371 has not been previously identified. The GNAS gene is located on chromosome 20q13.3 and encodes the $\alpha$ subunit of the heterotrimeric G protein complex (Gs $\alpha$ ), which couples seven-transmembrane receptors to the cAMP-generating enzyme adenylyl cyclase (8). Activating or inactivating mutations and epigenetic changes at the GNAS locus have been described in a number of cancers (9), but little is known regarding GNAS mutations in RCC. The findings of the present study must be replicated in a larger cohort and the mutations confirmed by additional methods. However, our data warrant further investigation into the role of GNAS mutation in RCC.

A recurrent mutation at Y55 was identified in the PTEN gene. Mutation of this residue has been previously identified in chromophobe RCC, uterine endometrioid carcinoma, glio- blastoma multiforme, breast invasive ductal carcinoma (IDC) and cervical squamous cell carcinoma (SCC), and is predicted to be oncogenic (7). It is well established that PTEN is a frequently mutated gene in RCC, particularly clear cell RCC (10). Moreover, several studies have demonstrated that mutations in PTEN are associated with worse overall survival in patients with RCC $(10,11)$. Therefore, it was not surprising to find a relatively high frequency of PTEN mutation in our cohort.

The RICTOR A3V mutation has been identified in breast IDC, and while the R8H mutation has not been previously identified, the R8C mutation has been identified in adenoid cystic carcinoma and hepatocellular carcinoma. RICTOR is the mTOR partner in the mTORC2 complex, which has been shown to serve an important function in the development and progression of RCC (12); thus, the identification of mutations in this gene are expected in RCC. None of the FGFR4 mutations have been previously identified in cancer, but the FGFR pathway is involved in driving VEGF-independent tumor angiogenesis as a mechanism to escape VEGF-targeted treatment (13). As several patients in our cohort had received such treatment, it is possible that these mutations arose in response to this treatment. The same may be the case for the mutation identified in FLT1, the gene that encodes VEGFR1. The AURKA mutation also has not been previously identified in cancer, but the role of Aurora kinase A has been described in advanced RCC (14), which may also be of relevance.

A subgroup analysis of patients who had been exposed to any MET inhibitor $(n=29)$ vs. those who had not $(n=21)$ was performed, and revealed that only patients who had been exposed to these agents had GAs in MET (n=6) and RET $(n=1)$; these GAs were not identified in any patients who had not received MET inhibitor treatment. The two tyrosine kinase-encoding genes RET and MET are targets of cabozantinib and other MET inhibitors (15), and RET mutations have been shown experimentally to cause resistance to cabozantinib in model systems (16), as have mutations in MET (17). Of the mutations in our cohort, the RET E366X mutation has been found in head and neck SCC and lung SCC. Mutation at aa63 has been found in uterine endometrioid carcinoma and mutation at aa375 has been found in prostate cancer, while the V1220I mutation has not been previously identified in cancer. It is possible that these mutations arose in response to treatment 
Table II. Comparison of mutation frequency across datasets.

\begin{tabular}{|c|c|c|c|c|}
\hline \multirow[b]{2}{*}{ Gene } & \multicolumn{4}{|c|}{ Samples with genomic alterations (\%) } \\
\hline & Present study & Pal et al & TCGA clear cell & TCGA papillary \\
\hline MET & 12 & 9.5 & 0.7 & 13 \\
\hline$M Y C$ & 16 & 7.7 & 1.2 & 0 \\
\hline PTEN & 20 & 3.6 & 5 & 2.9 \\
\hline TP53 & 4 & 35 & 2.1 & 2.5 \\
\hline GNAS & 28 & 3.2 & 0.7 & 0 \\
\hline HNF 1A & 8 & 0 & 0 & 1.5 \\
\hline FGFR4 & 6 & $\mathrm{~N} / \mathrm{S}$ & 0.7 & 0.7 \\
\hline RICTOR & 4 & $\mathrm{~N} / \mathrm{S}$ & 1 & 1.1 \\
\hline MTOR & 2 & 0 & 5.4 & 1.4 \\
\hline$A K T 1$ & 2 & 0.5 & 0.5 & 0 \\
\hline$R E T$ & 2 & 0.9 & 0.2 & 0 \\
\hline FLTI & 2 & $\mathrm{~N} / \mathrm{S}$ & 1.4 & 1.4 \\
\hline JAK3 & 2 & 0.9 & 1 & 0.4 \\
\hline$A U R K A$ & 2 & $\mathrm{~N} / \mathrm{S}$ & 0 & 0 \\
\hline$I D H I$ & 2 & 1.4 & 1.2 & 0.7 \\
\hline MYD88 & 2 & $\mathrm{~N} / \mathrm{S}$ & 3 & 0 \\
\hline
\end{tabular}

TCGA, The Cancer Genome Atlas; N/S, not sequenced.

with the MET inhibitors; however, we did not identify significant differences in response or time-to-progression in MET inhibitor-treated patients with or without mutations in MET and $R E T$, which may be attributed to the small sample size.

The present study was unable to confirm the findings of Pal et al (6), which demonstrated that GAs in several genes were more common among patients treated with multiple lines of treatment vs. those receiving only one line of treatment, but this was likely due to the smaller cohort and smaller number of mutations identified in the present study. Of note, GAs in GNAS were not found to be more frequent in patients receiving only one line of therapy, but the relevance of this finding is unclear.

Although the majority of studies extract ctDNA from plasma for sequencing, other body fluids, including the urine, also contain ctDNA. Urine has several advantages compared to blood, as it is a non-invasive sample source, which is ideal for patients who require repeated sampling to monitor cancer progression or therapeutic efficacy, and the collection of urine does not require specialized facilities or expensive equipment (18). Several studies have demonstrated that there is a high degree of correlation between the detection of specific GAs in the urine and plasma ctDNA $(19,20)$. We herein observed that, while there was a significant correlation between the number of GAs detected in matched urine and plasma samples, only $28.1 \%$ of GAs detected in plasma samples were also detected in matched urine samples. This may be due to technical complications from isolating ctDNA from urine (18), or it may reflect different subsets or clones of cancers that preferentially shed DNA into these fluids. Further research is needed to determine the association between ctDNA isolated from urine and plasma before urine ctDNA can reliably be used to monitor metastatic RCC.
Certain differences from the study of Pal et al (6) were to be expected, as a different sequencing platform was used and the patient population was also different. The present study included fewer patients, and they were all from a single institution. Furthermore, the treatment differed significantly between the two studies, as the patients in the Pal et al study received primarily VEGF-targeted therapies, while the treatments received by patients in the present study varied widely. The ctDNA sequencing platform used in the present study detected only mutations, not amplifications, which may partially explain why fewer GAs we identified in certain genes compared with the Pal et al study.

There were certain limitations to the present study. First, including only 50 patients from a single institution precludes wide generalizations of the findings, and it also limits subgroup analysis between different treatments and histological subtypes. The present study did not have primary tumor tissue to compare ctDNA samples, and serial samples were not collected, so it was not feasible to directly assess tumor evolution in individual patients. Furthermore, DNA from normal cells was not available for comparing ctDNA results; therefore, GAs were determined by comparison to a reference database. In addition, the investigational ctDNA sequencing panel used is marketed in China, but has not undergone testing as rigorous as that of its US-approved counterparts, such as those from Guardant or Foundation Medicine. Several of the GAs identified in the present study are variants of unknown significance and must be further investigated to determine whether they are truly oncogenic.

In conclusion, despite its limitations, the present study largely supports the findings of Pal et al and has identified potential new targets in metastatic RCC. 


\section{Acknowledgements}

The authors would like to thank the clinicians at City of Hope Medical Center for their help with enrolling patients in the present study.

\section{Funding}

The present study was funded by Beijing USCI Medical Laboratory.

\section{Availability of data and materials}

De-identified data are available from the corresponding author upon reasonable request.

\section{Authors' contributions}

JZ, YL, BX, FL, YW, ML, RD and YZ performed sample preparation, sequencing, data analysis and edited the manuscript. MS and LY collected and processed biological samples. JOJ assisted with sample processing, data analysis and manuscript preparation. All authors read and approved the final manuscript.

\section{Ethics approval and consent to participate}

The study protocol was approved by the City of Hope IRB and informed consent was obtained from all individual participants.

\section{Patient consent for publication}

Not applicable.

\section{Competing interests}

JZ, YL, BX, FL, YW, ML, RD and YZ are employees of Scisoon, a subsidiary of Beijing USCI Medical Laboratory. JOJ is a compensated advisor for Scisoon.

\section{References}

1. Ferlay J, Soerjomataram I, Dikshit R, Eser S, Mathers C, Rebelo M, Parkin DM, Forman D and Bray F: Cancer incidence and mortality worldwide: Sources, methods and major patterns in GLOBOCAN 2012. Int J Cancer 136: E359-E386, 2015.

2. Gupta K, Miller JD, Li JZ, Russell MW and Charbonneau C: Epidemiologic and socioeconomic burden of metastatic renal cell carcinoma (mRCC): A literature review. Cancer Treat Rev 34: 193-205, 2008

3. Cancer Genome Atlas Research Network, Linehan WM, Spellman PT, Ricketts CJ, Creighton CJ, Fei SS, Davis C, Wheeler DA, Murray BA, Schmidt L, et al: Comprehensive molecular characterization of papillary renal cell carcinoma. $\mathrm{N}$ Engl J Med 374: 135-145, 2016.

4. Cancer Genome Atlas Research Network: Comprehensive molecular characterization of clear cell renal cell carcinoma. Nature 499: 43-49, 2013.
5. Gainor JF, Dardaei L, Yoda S, Friboulet L, Leshchiner I, Katayama R, Dagogo-Jack I, Gadgeel S, Schultz K, Singh M, et al: Molecular mechanisms of resistance to first- and second-generation ALK inhibitors in ALK-rearranged lung cancer. Cancer Discov 6: 1118-1133, 2016.

6. Pal SK, Sonpavde G, Agarwal N, Vogelzang NJ, Srinivas S, Haas NB, Signoretti S, McGregor BA, Jones J, Lanman RB, et al: Evolution of circulating tumor DNA profile from first-line to subsequent therapy in metastatic renal cell carcinoma. Eur Urol 72: 557-564, 2017.

7. Cerami E, Gao J, Dogrusoz U, Gross BE, Sumer SO, Aksoy BA, Jacobsen A, Byrne CJ, Heuer ML, Larsson E, et al: The cBio cancer genomics portal: An open platform for exploring multidimensional cancer genomics data. Cancer Discov 2: 401-404, 2012.

8. Weinstein LS, Liu J, Sakamoto A, Xie T and Chen M: Minireview: GNAS: Normal and abnormal functions. Endocrinology 145: 5459-5464, 2004.

9. Furukawa T, Kuboki Y, Tanji E, Yoshida S, Hatori T, Yamamoto M, Shibata N, Shimizu K, Kamatani N and Shiratori K: Whole-exome sequencing uncovers frequent GNAS mutations in intraductal papillary mucinous neoplasms of the pancreas. Sci Rep 1: 161, 2011.

10. Fan C, Zhao C, Wang F, Li S and Wang J: Significance of PTEN mutation in cellular process, prognosis, and drug selection in clear cell renal cell carcinoma. Front Oncol 9: 357, 2019.

11. Que WC, Qiu HQ, Cheng Y, Liu MB and Wu CY: PTEN in kidney cancer: A review and meta-analysis. Clin Chim Acta 480: 92-98, 2018.

12. Sun B, Chen L, Fu H, Guo L, Guo H and Zhang N: Upregulation of RICTOR gene transcription by the proinflammatory cytokines through NF- $\kappa$ B pathway contributes to the metastasis of renal cell carcinoma. Tumor Biol 37: 4457-4466, 2016.

13. Massari F, Ciccarese C, Santoni M,Lopez-Beltran A, Scarpelli M, Montironi R and Cheng L: Targeting fibroblast growth factor receptor (FGFR) pathway in renal cell carcinoma. Expert Rev Anticancer Ther 15: 1367-1369, 2015.

14. Pal SK, He M, Tong T, Wu H, Liu X, Lau C, Wang JH, Warden C, Wu X, Signoretti S, et al: RNA-seq reveals aurora kinase-driven mTOR pathway activation in patients with sarcomatoid metastatic renal cell carcinoma. Mol Cancer Res 13: 130-137, 2015.

15. Osanto $\mathrm{S}$ and van der Hulle T: Cabozantinib in the treatment of advanced renal cell carcinoma in adults following prior vascular endothelial growth factor targeted therapy: Clinical trial evidence and experience. Ther Adv Urol 10: 109-123, 2018.

16. Liu X, Shen T, Mooers BHM, Hilberg F and Wu J: Drug resistance profiles of mutations in the RET kinase domain. Br J Pharmacol 175: 3504-3515, 2018.

17. Bahcall M, Sim T, Paweletz CP, Patel JD, Alden RS, Kuang Y, Sacher AG, Kim ND, Lydon CA, Awad MM, et al: Acquired METD1228V mutation and resistance to MET inhibition in lung cancer. Cancer Discov 6: 1334-1341, 2016.

18. Lu T and Li J: Clinical applications of urinary cell-free DNA in cancer: Current insights and promising future. Am J Cancer Res 7: 2318-2332, 2017.

19. Reckamp KL, Melnikova VO, Karlovich C, Sequist LV, Camidge DR, Wakelee H, Perol M, Oxnard GR, Kosco K, Croucher P, et al: A highly sensitive and quantitative test platform for detection of NSCLC EGFR mutations in urine and plasma. J Thorac Oncol 11: 1690-1700, 2016.

20. Fujii T, Barzi A, Sartore-Bianchi A, Cassingena A, Siravegna G, Karp DD, Piha-Paul SA, Subbiah V, Tsimberidou AM, Huang $\mathrm{HJ}$, et al: Mutation-enrichment next-generation sequencing for quantitative detection of KRAS mutations in urine cell-free DNA from patients with advanced cancers. Clin Cancer Res 23: 3657-3666, 2017

This work is licensed under a Creative Commons Attribution-NonCommercial-NoDerivatives 4.0 International (CC BY-NC-ND 4.0) License. 